\title{
Importance of Ultrasonography in Inspissated Bile Syndrome in a Neonate - A Case Report
}

\author{
Sharvari Shashikant Gulve ${ }^{1}$, Suresh Vasant Phatak ${ }^{2}$, Amruta Dinesh Varma ${ }^{3}$, Rishabh Surendra Gupta ${ }^{4}$
}

${ }^{1}$ Department of Radiodiagnosis, Datta Meghe Institute of Medical Sciences, Sawangi, Wardha, Maharashtra, India. 2Department of Radiodiagnosis, Datta Meghe Institute of Medical Sciences, Sawangi, Wardha, Maharashtra, India. ${ }^{3}$ Department of Radiodiagnosis, Datta Meghe Institute of Medical Sciences, Sawangi, Wardha, Maharashtra, India. ${ }^{4}$ Department of Radiodiagnosis, Datta Meghe Institute of Medical Sciences, Sawangi, Wardha, Maharashtra, India.

\section{INTRODUCTION}

Neonatal cholestasis is the diagnostic dilemma in clinical practice. ${ }^{1}$ It is a serious condition with the varied aetiological factors. Biliary atresia, hepatitis, choledochal cyst are common causes in neonates. Intrahepatic and extrahepatic causes can be easily differentiated by radiological investigations. ${ }^{2}$ Inspissated bile syndrome is a rare cause of neonatal jaundice. ${ }^{3,4}$ Predisposing factors for the development of inspissated bile in neonates include haemolysis, prematurity, parenteral nutrition, sepsis, and diuretic therapy. ${ }^{5}$ Ultrasonography is valuable in diagnosing the causes of obstructive jaundice especially in neonates where computed tomography scan cannot be done due to radiation exposure and MRI cannot be done due to the motion artefacts. Hepatobiliary scintigraphy single-photon emission computer tomography (HBS SPECT) is the most reliable diagnostic method. Generally, inspissated bile resolves spontaneously, but sometimes patient needs ursodeoxycholic acid. Rarely surgical drainage is also needed.

Inspissated bile syndrome is a rare cause of neonatal jaundice. It is extrahepatic obstruction due to sludge of inspissated bile. We present a case of 8 days old child who presented with jaundice. Total bilirubin was raised mainly due to high levels of unconjugated bilirubin.

\section{PRESENTATION OF CASE}

A 8 days old male child was to brought to the dept of radiodiagnosis for ultrasonography. The patient had yellow discoloration of skin and sclera. The patient was also had fever and tachycardia. It was full term vaginal delivery with assisted techniques. The patient didn't not cry immediately after birth and the had poor APGAR score at $1 \mathrm{~min}$. The patient also developed cephalohaematoma. On lab investigations total bilirubin levels were $16.2 \mathrm{mg} / \mathrm{dL}$, unconjugated bilirubin was $11.5 \mathrm{mg} / \mathrm{dL}$, and conjugated bilirubin was $4.7 \mathrm{mg} / \mathrm{dL}$. Child also had raised WBC levels (20600). Alkaline phosphate - 84, serum glutamate pyruvate transaminase (SGPT) - 26, serum glutamic oxaloacetic transaminase (SGOT) -61 and total protein5.5 and albumin - 3.3. Since the unconjugated bilirubin was raised liver disease was suspected and patient was brought to ultrasonography for further investigation.
Corresponding Author: Dr. Suresh Vasant Phatak, AVBRH Campus, DMIMS, Sawangi, Wardha. Maharashtra, India E-mail: suresh_phatak@yahoo.com

DOI: $10.14260 / \mathrm{jemds} / 2020 / 660$

How to Cite This Article:

Gulve SS, Phatak SV, Varma AD, et al. Importance of ultrasonography in inspissated bile syndrome in a neonate: $a$ case report. J Evolution Med Dent Sci 2020;9(40):3016-3018, $10.14260 / \mathrm{jemds} / 2020 / 660$

Submission 02-07-2020,

Peer Review 26-08-2020,

Acceptance 02-09-2020,

Published 05-10-2020.

Copyright (C) 2020 Sharvari Shashikant Gulve, et al. This is an open access article distributed under Creative Commons Attribution License [Attribution 4.0 International (CC BY 4.0)] 
Ultrasonography was done by using an $8-13 \mathrm{MHz}$ probe. On ultrasonography greyscale image, there was dilatation of common bile duct. In the dilated common bile duct there was echogenic material without any posterior shadowing which was suggestive of inspissated bile in it (Fig. 1). The common bile duct measured in $7 \mathrm{~mm}$ diameter, however Intra Hepatic Biliary Radicle were normal. Gall bladder was distended (Fig. 2). Wall thickness of gall bladder was normal. Liver measured $4.5 \mathrm{cms}$, with normal echotexture and the portal vain was also normal (Fig. 3). There was no e/o choledochal cyst, or extrahepatic biliary atresia. Bilateral kidneys and ureter were normal. Visualised bowel was normal. Later the patient was put on ursodeoxycholic acid.
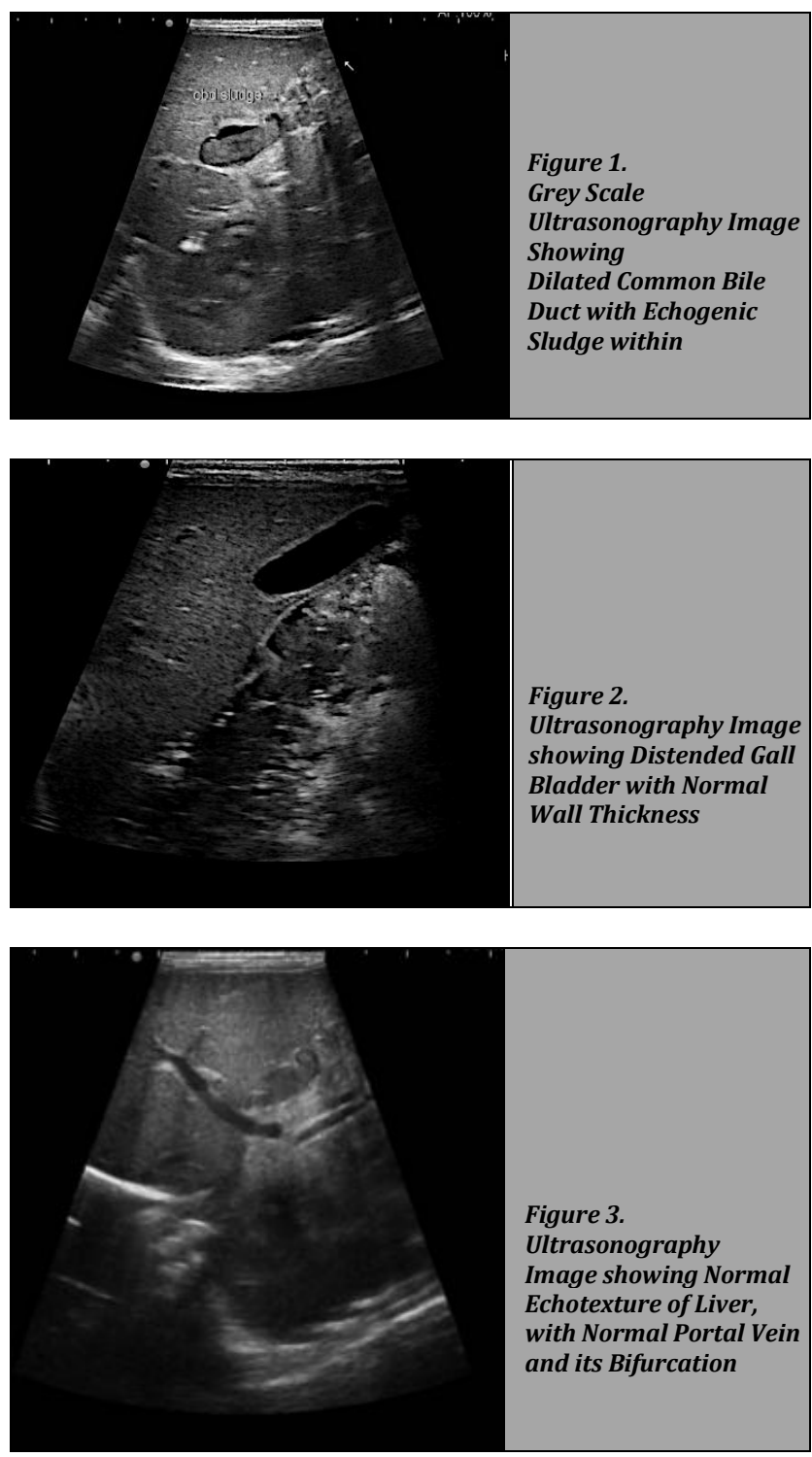

\section{DISCUSSION}

Cholestasis refers to decreased bile formation or excretion. Neonatal cholestasis is hyperbilirubinemia that is present at birth or develops in few days after birth. ${ }^{1}$

Cholestasis can cause liver dysfunction, liver transplantation may be required eventually leading to death. Thus, early diagnosis and evaluation are important. ${ }^{6}$ biliary atresia is most common cause of cholestasis in $1^{\text {st }}$ month of life. ${ }^{6}$ The various causes for obstructive jaundice in neonates includes, Choledochal cyst, Caroli disease and inspissated bile syndrome. ${ }^{7}$ The clinical symptom include acholic stool, jaundice, yellow pigmented urine and rarely bleeding due vit $\mathrm{k}$ deficiency secondary to cholestasis. In neonates differentiating between biliary atresia and neonatal cholestasis is crucial as biliary atresia requires surgery. ${ }^{8}$

Ultrasonography is well suited for the disease of gall bladder and liver in neonates as is free of radiations. ${ }^{9}$ Ultrasonography plays an important role in differentiating obstructive and non-obstructive causes of neonatal jaundice. ${ }^{7}$ Liver ultrasonography should be performed with 3 - 4 hours of fasting allowing the full distention of the gall bladder and the minimising the amount of the bowel gas. In -young infants higher frequency transducers of $7.5-12 \mathrm{MHz}$ are also helpful in young infants because of their small size. ${ }^{8}$ Normal liver size is $5-9.5 \mathrm{cms}$ and common bile duct diameter is $0.1-0.2 \mathrm{cms}{ }^{8}$ Hepatobiliary scintigraphy is generally used to differentiate biliary atresia from other causes of cholestasis by showing patency of biliary tract. If $5 \mathrm{mg} / \mathrm{kg} /$ day phenobarbital is started at least 5 days prior, biliary excretion of the isotope is enhanced but has delayed diagnosis. If uptake of the labelled substances by the hepatocytes is delayed, neonatal hepatitis should be considered. hepatobiliary scintigraphy singlephoton emission computer tomography (HBS SPECT) is most reliable diagnostic method compared to magnetic resonance cholangiography, HBS (hepatobiliary scintigraphy), or ultrasonography. Magnetic Resonance CholangioPancreatography is a noninvasive technique for evaluating the intrahepatic-extrahepatic bile ducts and the pancreatic duct. ${ }^{6}$ Magnetic Resonance imaging has advantages of multiplanar and multiphase imaging, high contrast resolution and lack of ionizing radiation, making it the preferred modality over computed tomography. ${ }^{8}$

Inspissated bile syndrome or bile plug syndrome is extrahepatic obstruction of bile duct by sludge in full term infants without any congenital abnormality or hepatocellular lesion. ${ }^{10}$ It is rare cause of jaundice in neonates. ${ }^{3,4}$ In such cases sludge in gallbladder appears as low-level echoes within the lumen. Inspissated bile is slightly more echogenic but does not give shadowing. Sometimes it is seen in common bile duct causing partial or complete biliary duct obstruction. Inspissated bile syndrome may be associated with massive haemolysis due to $\mathrm{Rh}$ incompatibility, haemorrhage or in various intestinal disease (increase enterohepatic circulation). ${ }^{4}$ other risk factors for formation of sludge are total parenteral nutrition, diuretic, cephalosporin and prematurity. ${ }^{8}$

Inspissated bile may resolve spontaneously or with ursodeoxycholic acid. Rarely endoscopic retrograde cholangiopancreatography or percutaneous drainage may be needed. ${ }^{8}$

Earlier complications like cholangitis and liver abscess are treated with percutaneous transhepatic cholangiography (PTC) or cholecystography with irrigation of the biliary tree with saline. ${ }^{2}$

\section{CONCLUSIONS}

Inspissated bile syndrome is rare cause of neonatal jaundice. Ultrasonography plays an important role in diagnosis of this 
rare condition, especially in neonates where investigation by other modalities is difficult. Other modalities that can be used for confirmation are hepatobiliary scintigraphy, single-photon emission computer tomography (HBS SPECT), hepatobiliary scintigraphy and magnetic resonance cholangiopancreatography. Hepatobiliary scintigraphy singlephoton emission computer tomography (HBS SPECT), is the most reliable investigation. Thus directing the clinician for proper treatment of the patient. Generally the bile plaque passes out spontaneous or with ursodeoxycholic acid. Rarely endoscopic retrograde cholangiopancreatography or percutaneous drainage has to be done.

Complications like Cholangitis and liver abscess may arise which has to be treated surgically.

Financial or Other Competing Interests: None.

\section{REFERENCES}

[1] Pandita A, Gupta V, Gupta G. Neonatal cholestasis: a Pandora's Box. Clin Med Insights Pediatr 2018; 12:1179556518805412.
[2] Matos MDR, Ferreira AT, Nunes AV, et al. Jaundice in children-imaging features. European Congress of Radiology ECR 2011.

[3] Gubernick JA, Rosenberg HK, Ilaslan H, et al. US approach to jaundice in infants and children. Radiographics 2000;20(1):173-95.

[4] Brownschidle S, Sullivan J, Sartorelli K, et al. Neonatal cholestasis due to biliary sludge-review and report of a case associated with use of diflucan. Ann Clin Path 2014;2(2):1018.

[5] Shah I. Neonatal cholestasis in an infant with $\mathrm{Rh}$ incompatibility. Pediatric Oncall J 2017;14(4):99-100.

[6] Gunaydin M, Cil ATB. Cholestasis in the baby and infant. EMJ 2019;4(3):73-82.

[7] Wani BN, Jajoo SN. Obstructive jaundice in neonates. Trop Gastroenterol 2009;30(4):195-200.

[8] Shamir SB, Kurian J, Kogan-Liberman D, et al. Hepatic imaging in neonates and young infants: state of the art. Radiology 2017;285(3):763-77.

[9] Haller JO. Sonography of the biliary tract in infants and children. AJR. Am J Roentgenol 1991;157(5):1051-8.

[10] Lang EV, Pinckney LE. Spontaneous resolution of bileplug syndrome. Am J Roentgenol 1991;156(6):1225-6. 